\title{
PTU-046 HOW COMMONLY IS THE DIAGNOSIS OF COELIAC DISEASE MADE BY SEROLOGICAL TESTS ALONE?
}

doi:10.1136/gut.2011.239301.174

G K T Holmes* Department of Gastroenterology, Derby Hospitals Foundation Trust, Derby, UK

Introduction While guidelines state that a small bowel biopsy is mandatory for the diagnosis of coeliac disease (CD) it is clear that doctors in Derby when confronted by an ill patient with positive coeliac serology or even an asymptomatic one with a positive test are often accepting this as diagnostic and not referring for small bowel biopsy. The present study aimed to determine how many patients in Derby are being given the diagnosis of CD based on serological tests alone.

Methods Records of the Derby hospital biochemical laboratory were used to identify all patients with positive coeliac serology (EMA) from 1996 to 2008. All those found were checked to determine whether a small bowel biopsy had been performed. This was achieved by reviewing hospital notes, checking the records of the histopathology department and making contact with general practitioners and patients. All patients were traced to determine their biopsy status. For these years all patients with biopsy prove CD in Derby were identified. Sources of information included the hospital diagnostic index, histopathology records, the dermatitis herpetiformis clinic and the dietetic department.

Results Table 1 shows that in the years 1996 to 2000, 29 patients ( $8 \%$ of the total) were not biopsied. The percentages for 2007 and 2008 were 46 and 53, respectively.

Table 1 PTU-046 Percentage of Patients with Coeliac Disease Diagnosed by Serology

\begin{tabular}{lclll}
\hline Years & Serological diag & Biopsy diag & Total diag & Serological \% \\
\hline $1996-2000$ & 29 & 335 & 364 & 8 \\
$2001-2006$ & 136 & 476 & 612 & 22 \\
2007 & 60 & 70 & 130 & 46 \\
2008 & 67 & 60 & 127 & 53 \\
\hline
\end{tabular}

Conclusion A positive serological test alone is being increasingly relied on to establish the diagnosis of CD mainly by General Practitioners but also by secondary care physicians. This is not unreasonable since serological tests are reliable ${ }^{1}$ and guidelines need to be reviewed.

Competing interests None.

Keywords Coeliac disease, Diagnosis, Serological tests.

\section{REFERENCE}

1. Hill PG, Holmes GKT. Coeliac disease: a biopsy is not always necessary for diagnosis. Aliment Pharmacol Ther 2008;27:572-77. 